\title{
The Dihydrofolate Reductase Protein-Fragment Complementation Assay: A Survival-Selection Assay for Large-Scale Analysis of Protein-Protein Interactions
}

\author{
Stephen W. Michnick, ${ }^{1,5}$ Emmanuel D. Levy, ${ }^{2,5}$ Christian R. Landry, ${ }^{3,5}$ Jacqueline Kowarzyk, ${ }^{1}$ \\ and Vincent Messier ${ }^{1,4}$ \\ ${ }^{1}$ Département de Biochimie et Médecine Moléculaire, Université de Montréal, Montréal, Québec H3C 3J7, \\ Canada; ${ }^{2}$ Department of Structural Biology, Weizmann Institute of Science, Rehovot 76100 Israel; ${ }^{3}$ Département \\ de Biologie, Institut de Biologie Intégrative et des Systèmes, PROTEO-Québec Research Network on Protein \\ Function, Structure and Engineering, Université Laval, Québec, Québec G1V 0A6, Canada; ${ }^{4}$ Department of \\ Medical Research, University of Toronto, Toronto, Ontario M5S 3E1, Canada
}

Protein-fragment complementation assays (PCAs) can be used to study protein-protein interactions (PPIs) in any living cell, in vivo or in vitro, in any subcellular compartment or membranes. Here, we present a detailed protocol for performing and analyzing a high-throughput PCA screening to study PPIs in yeast, using dihydrofolate reductase (DHFR) as the reporter protein. The DHFR PCA is a simple survival-selection assay in which Saccharomyces cerevisiae DHFR (scDHFR) is inhibited by methotrexate, thus preventing nucleotide synthesis and causing arrest of cell division. Complementation of cells with a methotrexate-insensitive murine DHFR restores nucleotide synthesis, allowing cell proliferation. The methotrexate-resistant DHFR has two mutations (L22F and F31S) and is 10,000 times less sensitive to methotrexate than wild-type scDHFR, but retains full catalytic activity. The DHFR PCA is sensitive enough for PPIs to be detected for open reading frame (ORF)-PCA fragments expressed off of their endogenous promoters.

It is essential that you consult the appropriate Material Safety Data Sheets and your institution's Environmental Health and Safety Office for proper handling of equipment and hazardous materials used in this protocol.

RECIPES: Please see the end of this protocol for recipes indicated by $<R>$. Additional recipes can be found online at http://cshprotocols.cshlp.org/site/recipes.

Reagents

Commercial bleach solution (10\%) (4\% sodium hypochloride)

Dimethylsulfoxide (DMSO)

Glycerol solution (75\%)

Sterilize by autoclaving for $30 \mathrm{~min}$ at $121^{\circ} \mathrm{C}$.

\footnotetext{
${ }^{5}$ Correspondence: stephen.michnick@umontreal.ca; emmanuel.levy@weizmann.ac.il; christian.landry@bio.ulaval.ca 
S.W. Michnick et al.

TABLE 1. Plasmid and oligonucleotide list (Tarassov et al. 2008)

\begin{tabular}{|c|c|}
\hline Plasmid or oligonucleotide & Characteristics \\
\hline $\begin{array}{l}\text { pAG25-linker-DHFR-F[1,2] } \\
\text { Antibiotic resistance: nourseothricin } \\
\text { pAG32-linker-DHFR-F[3] } \\
\text { Antibiotic resistance: hygromycin-B }\end{array}$ & $\begin{array}{l}\text { For creating integration cassettes of DHFR PCA fragments to study yeast PPIs at } \\
\text { endogenous levels of expression. Cassettes are integrated by homologous } \\
\text { recombination. }\end{array}$ \\
\hline $\begin{array}{l}\text { Forward oligonucleotides for creating } \\
\text { integration cassettes }\end{array}$ & $\begin{array}{l}61 \text { base pairs long, consisting of a } 40 \text {-nucleotide sequence homologous to a } \\
\text { region located } 5^{\prime} \text { to the ORF stop codon, followed by a } 21 \text {-nucleotide sequence } \\
\text { that anneals to the linker: } 5^{\prime} \text {-GGCGGTGGCGGATCAGGAGGC-3' }\end{array}$ \\
\hline $\begin{array}{l}\text { Reverse oligonucleotides for creating } \\
\text { integration cassettes }\end{array}$ & $\begin{array}{l}64 \text { base pairs long, consisting of a } 40 \text {-nucleotide sequence homologous to the } \\
\text { sequence of genomic DNA immediately } 3^{\prime} \text { to the stop codon of each ORF, } \\
\text { followed by a } 24 \text {-nucleotide sequence that anneals to the } 3^{\prime} \text { end of the TEF } \\
\text { terminator region of the antibiotic resistance cassettes: } 5^{\prime} \text {-TTCGACACTGGA } \\
\text { TGGCGGCGTTAG-3' }\end{array}$ \\
\hline
\end{tabular}

Oligonucleotides for amplification of protein-fragment complementation assay (PCA) fragment cassettes

Forward and reverse oligonucleotides for creation of homologous recombination cassettes are available for most genes on request. Should this not be the case, they can be designed following the specifications provided in Table 1.

Polymerase chain reaction (PCR) reagents

Plasmids containing the dihydrofolate reductase protein-fragment complementation assay (DHFR PCA) fragments, followed by a terminator and an antibiotic selection marker (Table 1) pAG25-linker-DHFR-F[1,2] pAG32-linker-DHFR-F[3]

We also have DHFR expression plasmids which allow bait-coding sequences to be modified with mutations and screened against prey arrays. These plasmids can be requested from the authors.

PLATE solution $<\mathrm{R}>$

Yeast culture media

Synthetic complete (SC) medium, minus adenine, plus methotrexate $(35 \mathrm{~mL}$ in OmniTrays $)<\mathrm{R}>$ Synthetic complete (SC) medium, minus lysine and methionine, plus antibiotics $(35 \mathrm{~mL}$ in

OmniTrays) (optional; see Step 18) $<\mathrm{R}>$

YPD for the DHFR PCA (solidified with $2 \%$ agar $[\mathrm{w} / \mathrm{v}]$ in Petri dishes with suitable antibiotic for the competent strain; Table 1) $<\mathrm{R}>$

YPD for the DHFR PCA (solidified with $3 \%$ agar $[\mathrm{w} / \mathrm{v}] ; 35 \mathrm{~mL}$ in OmniTrays) $<\mathrm{R}>$

For prey strain selective medium (Step 11), prepare with $100 \mu \mathrm{g} / \mathrm{mL}$ nourseothricin (for MATa recombinant strains) or $250 \mu \mathrm{g} / \mathrm{mL}$ hygromycin B (for MATa recombinant strains). For diploid selective medium (Step 17), prepare with both $100 \mu \mathrm{g} / \mathrm{mL}$ nourseothricin and $250 \mu \mathrm{g} / \mathrm{mL}$ hygromycin B.

YPD for the DHFR PCA (without agar) $<\mathrm{R}>$

For bait strain selective medium (Step 8), prepare with $100 \mu \mathrm{g} / \mathrm{mL}$ nourseothricin (for MATa recombinant

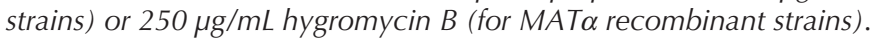

Yeast strains (competent, for use in Step 2)

BY4741 recombinant strains (MATa; his3 $\Delta 1$; leu2 $\Delta 0$; met15 0 ; ura3 $\Delta 0$ ) containing ORFs of interest (Open Biosystems) (to be fused to the complementary DHFR F[1,2] PCA fragment in Step 2)

BY4742 recombinant strains (MAT $\alpha$ his $3 \Delta 1$; leu2 $\Delta 0$; lys $2 \Delta 0$; ura $3 \Delta 0)$, containing ORFs of interest (Open Biosystems) (to be fused to the complementary DHFR F[3] PCA fragment in Step 2)

Here, we use MATa strains as bait and MAT $\alpha$ as prey strains; however, MATa or MAT $\alpha$ strains can be used as bait or prey as desired. Competent BY4741 and BY4742 strains can be used to generate new strains with ORFs tagged with DHFR PCA fragments, if strains containing the ORF of interest are not available at Open Biosystems.

Equipment

Bench centrifuge (Eppendorf 5810R)

Centrifuge tubes 
Glass beads

Incubator at $30^{\circ} \mathrm{C}$

OmniTray single-well plates (Nunc CA62409-600)

PCR equipment

Pintools, robotically or manually manipulated (V\&P Scientific)

Manually manipulated

96-pintool with $1.58-\mathrm{mm}, 1-\mu \mathrm{L}$ slot pins (VP 408Sa)

Robotically manipulated

96-pintool with $0.787-\mathrm{mm}$ flat round-shaped pins (FP3N)

384-pintool with $0.457-\mathrm{mm}$ flat round-shaped pins (custom FP1N)

1536-pintool with $0.457-\mathrm{mm}$ flat round-shaped pins (custom FP1N)

Plate imaging equipment

Camera (at least 4.0 megapixels, e.g., Canon Powershot A520)

Plate-shooting platform

We recommend covering the plate fixation platform with a black velvet cover.

Stationary arm (70-cm mini repro [Industria Fototecnica Firenze, Italy])

Shaking incubator at $30^{\circ} \mathrm{C}$

Water bath or heat block at $42^{\circ} \mathrm{C}$

METHOD

Fusing PCA Fragments at Chromosomal Loci by Homologous Recombination

1. PCR-amplify the PCA fragment cassettes from the pAG25 (DHFR fragment $[1,2]$ ) and pAG32 (DHFR fragment [3]) plasmids using forward and reverse oligonucleotides (Table 1).

2. Transform the appropriate strain of competent cells (BY4741 or BY4742 recombinant strains containing the ORFs of interest, according to experimental design) with each PCR product.

i. Mix $20 \mu \mathrm{L}$ of thawed competent cells with $8 \mu \mathrm{L}(\sim 1 \mu \mathrm{g} / \mu \mathrm{L})$ of each amplified cassette DNA (encoding the PCA fragments and a resistance marker).

ii. Add $100 \mu \mathrm{L}$ of PLATE solution.

iii. Incubate for $30 \mathrm{~min}$ at room temperature.

iv. Add $15 \mu \mathrm{L}$ DMSO and heat shock the cells for $15 \mathrm{~min}$ at $42^{\circ} \mathrm{C}$.

3. Centrifuge the cells at $805 \mathrm{~g}$ for $3 \mathrm{~min}$ at room temperature. Remove the supernatant and resuspend the cells in $100 \mu \mathrm{L}$ of YPD medium. Incubate with shaking for $4 \mathrm{~h}$ at $30^{\circ} \mathrm{C}$.

4. Centrifuge the cells at $805 \mathrm{~g}$ for $3 \mathrm{~min}$ at room temperature. Remove the supernatant and resuspend the cells in $200 \mu \mathrm{L}$ of YPD medium.

5. Plate the entire $200 \mu \mathrm{L}$ on a Petri dish containing solid YPD medium plus the suitable antibiotic (Table 1).

6. Incubate the plates for $48-72 \mathrm{~h}$ at $30^{\circ} \mathrm{C}$.

Colonies can be further verified using colony PCR.

7. Prepare glycerol stocks of the bait/prey strains for storage.

Performing a Large-Scale DHFR PCA Screen

In the following procedure, an array of "prey" strains is generated and mated to individual "bait" strains of the opposite mating type. Resulting diploids are transferred to methotrexate selection plates for the DHFR PCA (Fig. 1).

It is critical to use both positive and negative controls (baits and preys that are known to directly interact with each other, preferably as shown by multiple methods, as well as baits and preys that have never been shown to interact with each other) to assure the quality of the entire printing and selection process, and to allow the detection of grid 
A<smiles>C=CC(=O)N[C@@H](CCC(=O)O)C(=O)O</smiles><smiles>C=CCO</smiles>
Tetrahydrofolic acid (THF)

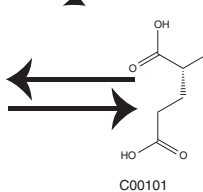

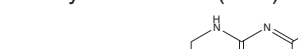

Nucleotide metabolism

B
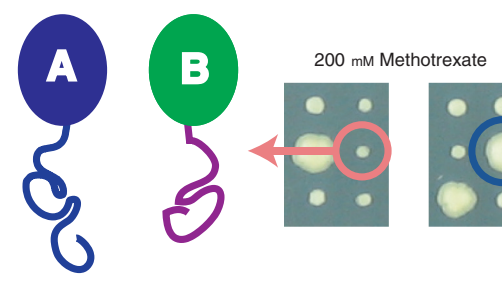

C

Bait strain liquid culture

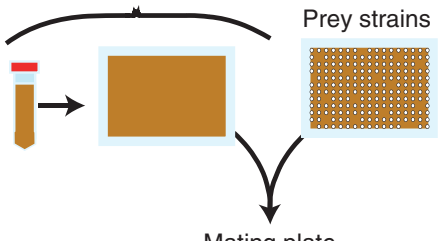

YPD + agar
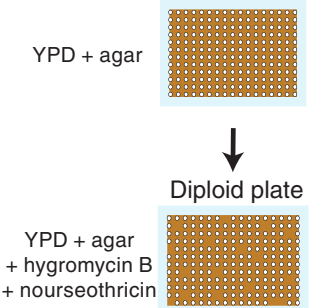

+ nourseothricin

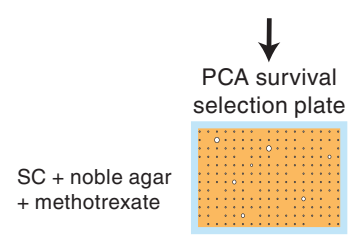

FIGURE 1. The DHFR PCA. (A) DHFR catalyzes the reduction of dihydrofolate to tetrahydrofolate, an early step in the synthesis of purine. Methotrexate is a potent and highly specific competitive inhibitor of DHFR. (B) Bait and prey proteins are fused to complementary fragments of methotrexate-insensitive murine DHFR. No DHFR PCA selection is observed if there is no bait-prey interaction. If there is a bait-prey interaction, DHFR activity is reconstituted, allowing cells to proliferate in the presence of methotrexate. $(C)$ Schematic representation of a DHFR PCA screen. The bait strain is incubated in liquid culture and the prey reporter strains are printed on solid medium. A mating plate is created through sequential printing of a bait to the prey strain array grown on agar in nonselection medium. Haploids and diploid mixture strains are transferred to agar plates containing a medium for the selection of diploids. The resulting diploid array is then transferred onto DHFR PCA selection plates containing methotrexate.

mispositioning or batch effects (e.g., differences in media, incubation conditions, or drug concentration) that could change growth rates of colonies on different plates; see Discussion.

\section{Preparing Bait Strains}

8. Pick the bait strains from thawed glycerol stocks and add the cells to $100 \mathrm{~mL}$ of bait strain selective medium.

9. Allow the resulting culture to reach saturation by shaking at $30^{\circ} \mathrm{C}$.

It is very important to obtain a saturated bait culture to print enough cells for efficient mating on solid phase. See Troubleshooting.

10. Centrifuge the saturated culture at $500 \mathrm{~g}$ for $5 \mathrm{~min}$. Resuspend the cells in $15 \mathrm{~mL}$ of YPD medium.

\section{Preparing Prey Strains}

11. Pick the prey strains from thawed glycerol stocks and print onto OmniTray plates containing 35 $\mathrm{mL}$ of solid prey strain selective medium. Clean the pintool between each cell transfer by soaking the pins twice in a $10 \%$ bleach solution containing glass beads, and then washing once in $10 \%$ bleach followed by twice in a sterile water bath.

Either 96-pin manual or robotic pintools can be used to print the prey array, with a total of 384 prints per plate. Step 11 can be repeated from the 384-prints to transfer the prey strain to a maximum of four other 1536-prints per OmniTray, achieving a density of up to 6144 colonies. 
12. Incubate the plates for $16 \mathrm{~h}$ at $30^{\circ} \mathrm{C}$.

See Troubleshooting.

\section{Performing the PCA Screen}

13. Transfer the bait strain suspensions into an empty OmniTray plate.

14. Print the bait strain suspensions from the OmniTray to another OmniTray containing $35 \mathrm{~mL}$ of solid YPD medium using an appropriate pintool for the desired colony array density.

15. Transfer the prey strains onto the bait strain using the same density pintool as used for the bait strain.

16. Allow mating to occur by incubating the plate for $16 \mathrm{~h}$ at $30^{\circ} \mathrm{C}$.

17. Transfer the mixed haploid and diploid colonies to an OmniTray plate containing $35 \mathrm{~mL}$ of solid diploid selective medium using an appropriate pintool for the desired colony array density.

18. Incubate the OmniTray for $48 \mathrm{~h}$ at $30^{\circ} \mathrm{C}$.

To increase the diploid selection stringency, mixed haploid and diploid colonies can be transferred onto SC medium, minus lysine and methionine, plus antibiotics (both nourseothricin and hygromycin B when using BY4741 MATa and BY4742 MAT $\alpha$ ). Stringency can also be increased by repeating Step 17.

See Troubleshooting.

19. Wet the pintool pin tips with $75 \%$ glycerol solution.

Liquid transfer with pintools is sensitive to the retraction speed of the pintool from the liquid, with faster speeds yielding more liquid on the pins. To obtain a homogeneous glycerol microdrop on every pin, the immersion depth and retraction speed may vary with each robotic setup and will need to be optimized, as it depends on several factors, such as the time between the last water wash and the glycerol dip, the room humidity, and the room air-flow.

20. Transfer the diploid selected strains onto an OmniTray plate containing $35 \mathrm{~mL}$ of solid SC medium, minus adenine, plus methotrexate, using an appropriate pintool for the desired colony array density.

SC medium without adenine is used to decrease background growth.

21. Incubate the OmniTray at $30^{\circ} \mathrm{C}$. Acquire pictures of the colony array every $24-96 \mathrm{~h}$ for up to $2 \mathrm{wk}$. See Troubleshooting.

\section{Analyzing Images of Large-Scale DHFR PCA Screens}

22. Measure the number of pixels per colony position on the selection plates.

Several bioinformatics tools are available to perform colony size measurements from digital images of high-density plates (Collins et al. 2006; Memarian et al. 2007). When densities up to 1536 colonies per plate are used, macros can be implemented in Image/ (http://rsb.info.nih.gov/ij/) or any other image analysis software. We convert digital images of plates to 8-bit gray scale format and measure colonies by positioning the measurement tool on a colony center and estimating integrated pixel intensity in an area corresponding to a maximal allowed colony size. We iterate this process over all grid positions and then over all of the plates. Colonies should always be found at the same positions in the images. If this is not so, a step can be included to position an analysis grid onto colony positions before measuring the colony sizes. Image analysis should be performed only on the region containing colonies by removing the plate sides from analysis. Images can be corrected for nonuniform illumination with algorithms such as those described here: http://blogs.mathworks.com/steve/2011/09/27/digital-image-processing-using-matlabreading-image-files/?s_tid=srchtitle. Artifactual objects such as bubbles, gel background, and other anomalies can be removed using the imopen function. When working with high-density plates (e.g., a 1536position grid or greater), methods must be used to segment adjacent and overlapping colonies (Tarassov et al. 2008).

Grid positions and intensity values can be saved as text files for processing in your favorite spreadsheet or statistical analysis software. 
S.W. Michnick et al.

\title{
Performing Statistical Analyses of Colony Data
}

Both positive and negative controls should be analyzed on every plate; see Discussion.

23. Perform statistical analyses to determine whether there is a significant difference in growth rates among the plates.

Significant variation in the data requires that it be normalized so that all of the plates have the same average colony size. Alternatively, relative scores of the data can be calculated (e.g., Z-scores) so that data points are transformed into the number of standard deviations separating the data point from the average value of an individual plate. We have combined the Z-score and the raw intensity for best interpretation of our largescale screens (Tarassov et al. 2008).

24. Convert continuous values into binary values by setting a threshold of intensity above which proteins are inferred to interact. Calculate the positive predictive value (PPV) as a function of threshold of intensities as follows:

$$
\mathrm{PPV}=\frac{\text { True positive interactions }}{\text { Total number of inferred positives }},
$$

Total number of inferred positives $=$ True positives + Predicted false positives.

\begin{abstract}
For example, at PPV $=95 \%, 5 \%$ of positives are predicted to be false. Thresholds are arbitrary, depending on the stringency one wants to achieve in the analysis. The relative occurrence of positives and negatives in the reference sets must be similar to that of the real positives and negatives to assure that estimated PPV values are accurate (Jansen and Gerstein 2004). For comprehensive genome-wide screens, this fraction is a very low prior probability of finding interactions among all possible pairs of proteins. In small-scale screen of a specific biological process, however, there will be a greater proportion of true positives than in a random screen. Thus, the choice of reference sets must be appropriate to the specific screen, specifically to the size of the potential interactome that will be covered.
\end{abstract}

25. Establish a confidence score for the selected threshold.

Confidence values can be applied to PCA interactions by benchmarking observed intensity values to the results of interactions that should be detected in the screen (a set of real positives) compared with interactions that should not be observed under any circumstances (e.g., a set of real negatives representing proteins that are expressed in different cellular compartments and never observed to interact) (Collins et al. 2007). For a given intensity threshold, one can then determine what should be the proportion of true positive and false positive interactions.

26. Perform a statistical analysis to separate interacting pairs from noninteracting pairs.

27. Perform Gene Ontology enrichments and visualize interaction clusters to further assess confidence in the produced data set.

Choosing true positives and negatives does not guarantee that observed interactions are functionally relevant. Only direct functional assays and evolutionary characterization of interactions are sufficient to determine whether an interaction is functionally relevant to an organism (Levy et al. 2009; Landry et al. 2013).

Problem (Steps 9, 12): Strains are not growing, or prey array growth is incomplete.

Solution: Verify the appropriate culture conditions, because the problem might be caused by erroneous haploid selection. If the problem is caused by low glycerol viability, strains can be streaked on solid agar-selective medium in Petri dishes before inoculation to increase viability. Finally, verify that all pins of the pintool touch the glycerol stocks and the recipient OmniTray plate.

Problem (Step 18): There are few or no colonies on diploid selective plates.

Solution: Verify the mating type of the haploid strains, because the problem might be caused by erroneous haploid strain type. The problem may also be technical, that is, pintool alignment 
might have changed. No modifications to the pintool positioning should be done between transfers.

Problem (Step 21): There is no colony growth on DHFR PCA survival-selective medium.

Solution: This problem can be caused by erroneous selection conditions. Use the heteromeric complex $\mathrm{SspB}_{\mathrm{YGMF}}: \mathrm{SspB}_{\mathrm{LSLA}}$ as a positive control to validate DHFR PCA activity (Tarassov et al. 2008). The problem might also be caused by an erroneous DHFR PCA. Perform a strain diagnostic PCR of the complementary PCA fragments and verify DHFR PCA fragment recombinant insertion by genomic sequencing.

Problem (Step 21): All colonies on the DHFR PCA survival-selective medium grow at the same rate.

Solution: Verify DHFR PCA fragment expression by western blot. The problem might be caused by erroneous selection conditions; use DHFR PCA fragment controls alone as negative control. Also verify methotrexate solubility under the conditions used. Stock solutions of methotrexate should not exceed $10 \mathrm{mg} / \mathrm{mL}$ in DMSO, and the final concentration in solid agar plates should not exceed $200 \mu \mathrm{g} / \mathrm{mL}$.

In the above protocol, the growth rates of the positive controls provide a rough indication of the intensity threshold that can be expected for strains expressing interacting bait-prey pairs of similar abundance level. Other critical controls are those for spontaneous PCA-cases in which a proteinPCA fragment fusion interacts with the complementary fragment alone (Tarassov et al. 2008). Additional controls can be used to examine the range of dissociation constants for which the DHFR PCA is sensitive (Campbell-Valois et al. 2005) and to test a condition-dependent PPI (Pelletier et al. 1998). A potential source of false positives in a PCA screen is the trapping of nonspecific complexes caused by irreversible folding of the DHFR fragments. However, we have used adenosine $3^{\prime}, 5^{\prime}$-monophosphate-dependent dissociation of the yeast protein kinase A complex (Michnick et al. 2007) to show that the DHFR PCA is fully reversible, and thus the trapping of complexes is unlikely (Tarassov et al. 2008). All of these reagents are available from the authors on request.

In an initial study, Freschi et al. (2013) showed that genetic or environmental perturbations that affect the interaction between the catalytic and regulatory subunits of PKA yield different growth rates when probed with the DHFR PCA (Freschi et al. 2013), indicating that the DHFR PCA is sensitive to the amount of complex formed, with a higher complex concentration leading to a faster growth. We characterized the extent and precision of this relationship in our recent experiment measuring the interactions between (i) a neutral reporter comprising the venus yellow fluorescent protein (YFP) fused to DHFR F[1,2] and (ii) the Mat $\alpha$ library of ORFs fused to DHFR[3], denoted $P(i)$, with $i=1, \ldots$, $N$ (with $N$ corresponding to up to 4808 interactions that can be examined) (Levy et al. 2014). Given that the concentration of YFP is constant, and that YFP only shows weak interactions of comparable $K_{d}$ with all yeast proteins, the main determinant of complex concentration [YFP $\cdot P(i)$ ] is the concentration of $P(i)$. Colony size measurements in this experiment enabled us to infer $[P(i)]$ with an accuracy comparable with that of mass spectrometry, demonstrating that the DHFR PCA assay is highly quantitative in determining the amount of complex formed. At the same time, this highlights the importance of controlling for protein abundance in a DHFR PCA, as a protein can yield a strong signal because of abundance alone. For example, two proteins present in the cell at nanomolar concentrations and showing a strong (nanomolar) $K_{a}$ would yield fewer complexes (and therefore lesser growth) than two proteins present at micromolar concentration and showing a weak (e.g., micromolar) $K_{a}$. 
Amino Acid Mix (10x)

Amino acid or nucleobase Concentration $(10 \times)$

Adenine sulfate

$0.4 \mathrm{~g} / \mathrm{L}$

Uracil

$0.2 \mathrm{~g} / \mathrm{L}$

L-Tryptophan

$0.4 \mathrm{~g} / \mathrm{L}$

L-Histidine hydrochloride

$0.2 \mathrm{~g} / \mathrm{L}$

L-Arginine hydrochloride

$0.2 \mathrm{~g} / \mathrm{L}$

L-Tyrosine

$0.3 \mathrm{~g} / \mathrm{L}$

L-Leucine

$0.6 \mathrm{~g} / \mathrm{L}$

L-Lysine hydrochloride

$0.3 \mathrm{~g} / \mathrm{L}$

L-Phenylalanine

$0.5 \mathrm{~g} / \mathrm{L}$

L-Glutamic acid

$1.0 \mathrm{~g} / \mathrm{L}$

L-Asparagine

$1.0 \mathrm{~g} / \mathrm{L}$

L-Valine

$1.5 \mathrm{~g} / \mathrm{L}$

L-Threonine

$2.0 \mathrm{~g} / \mathrm{L}$

L-Serine

$3.75 \mathrm{~g} / \mathrm{L}$

L-Methionine

$0.2 \mathrm{~g} / \mathrm{L}$

Dissolve the amino acids from the list above (except those to be excluded from any dropout media) in distilled $\mathrm{H}_{2} \mathrm{O}$. Filter-sterilize, store at $4^{\circ} \mathrm{C}$, and protect from light.

\section{PLATE Solution}

Reagents

Final concentration $(1 \times)$

PEG 3350

$40 \%(\mathrm{w} / \mathrm{v})$

Lithium acetate

$100 \mathrm{~mm}$

Tris $(\mathrm{pH} 7.5)$

$10 \mathrm{~mm}$

EDTA

$0.4 \mathrm{~mm}$

Wear personal protective equipment when handling this reagent. Store at room temperature in a dry and well-ventilated place.

Synthetic Complete Medium (SC), Minus Lysine and Methionine, Plus Antibiotics

Reagents

Final concentration $(1 \times)$

Yeast nitrogen base (YNB) medium without $1.74 \mathrm{~g} / \mathrm{L}$ amino acids or ammonium sulfate (Bioshop)

Noble agar (purified agar; Bioshop)

$4 \%(\mathrm{w} / \mathrm{v})$

$2 \%(\mathrm{w} / \mathrm{v})$

Amino acid mix (10×), without lysine or

$$
1 \times
$$

methionine $<\mathrm{R}>$

Nourseothricin (Jena Bioscience)

Hygromycin B (Wisent Bioproducts)

$100 \mu \mathrm{g} / \mathrm{mL}$

$250 \mu \mathrm{g} / \mathrm{mL}$

Synthetic Complete (SC) Medium, Minus Adenine, Plus Methotrexate

Reagents

Final concentration $(1 \times)$

Yeast nitrogen base (YNB) medium without $1.74 \mathrm{~g} / \mathrm{L}$ amino acids or ammonium sulfate (Bioshop)

Noble agar (purified agar; Bioshop)

Glucose solution (sterile)

Methotrexate

$2 \%(\mathrm{w} / \mathrm{v})$

Amino acid mix $(10 \times)$, without adenine $<\mathrm{R}>$ 
YPD for the DHFR PCA

Reagents

Yeast extract

Peptone

Dextrose

Agar

Store for up to several months at $4^{\circ} \mathrm{C}$.
Final concentration $(1 \times)$

$1 \%(\mathrm{w} / \mathrm{v})$

$2 \%(\mathrm{w} / \mathrm{v})$

$2 \%(\mathrm{w} / \mathrm{v})$

$2 \%(\mathrm{w} / \mathrm{v})$

\section{REFERENCES}

Campbell-Valois FX, Tarassov K, Michnick SW. 2005. Massive sequence perturbation of a small protein. Proc Natl Acad Sci 102: 14988-14993.

Collins SR, Schuldiner M, Krogan NJ, Weissman JS. 2006. A strategy for extracting and analyzing large-scale quantitative epistatic interaction data. Genome Biol 7: R63.

Collins SR, Kemmeren P, Zhao XC, Greenblatt JF, Spencer F, Holstege FC, Weissman JS, Krogan NJ. 2007. Toward a comprehensive atlas of the physical interactome of Saccharomyces cerevisiae. Mol Cell Proteomics 6: $439-450$

Freschi L, Torres-Quiroz F, Dube AK, Landry CR. 2013. qPCA: A scalable assay to measure the perturbation of protein-protein interactions in living cells. Mol BioSyst 9: 36-43.

Jansen R, Gerstein M. 2004. Analyzing protein function on a genomic scale: The importance of gold-standard positives and negatives for network prediction. Curr Opin Microbiol 7: 535-545.

Landry CR, Levy ED, Abd Rabbo D, Tarassov K, Michnick SW. 2013. Extracting insight from noisy cellular networks. Cell 155: 983-989.
Levy ED, Landry CR, Michnick SW. 2009. How perfect can protein interactomes be? Sci Signal 2: pe11.

Levy ED, Kowarzyk J, Michnick SW. 2014. High-resolution mapping of protein concentration reveals principles of proteome architecture and adaptation. Cell Rep 7: 1333-1340.

Memarian N, Jessulat M, Alirezaie J, Mir-Rashed N, Xu J, Zareie M, Smith M, Golshani A. 2007. Colony size measurement of the yeast gene deletion strains for functional genomics. BMC Bioinformatics 8: 117.

Michnick SW, Ear PH, Manderson EN, Remy I, Stefan E. 2007. Universal strategies in research and drug discovery based on protein-fragment complementation assays. Nat Rev 6: 569-582.

Pelletier JN, Campbell-Valois FX, Michnick SW. 1998. Oligomerization domain-directed reassembly of active dihydrofolate reductase from rationally designed fragments. Proc Natl Acad Sci 95: 12141-12146.

Tarassov K, Messier V, Landry CR, Radinovic S, Serna Molina MM, Shames I, Malitskaya Y, Vogel J, Bussey H, Michnick SW. 2008. An in vivo map of the yeast protein interactome. Science 320: 1465-1470. 


\section{The Dihydrofolate Reductase Protein-Fragment Complementation Assay: A} Survival-Selection Assay for Large-Scale Analysis of Protein-Protein Interactions

Stephen W. Michnick, Emmanuel D. Levy, Christian R. Landry, Jacqueline Kowarzyk and Vincent Messier

Cold Spring Harb Protoc; doi: 10.1101/pdb.prot090027

\begin{tabular}{|c|c|}
\hline $\begin{array}{l}\text { Email Alerting } \\
\text { Service }\end{array}$ & Receive free email alerts when new articles cite this article - click here. \\
\hline $\begin{array}{l}\text { Subject } \\
\text { Categories }\end{array}$ & $\begin{array}{l}\text { Browse articles on similar topics from Cold Spring Harbor Protocols. } \\
\text { Cell Biology, general (1382 articles) } \\
\text { High-throughput Analysis of Protein:Protein Interactions (39 articles) } \\
\text { Protein: Protein Interactions ( } 86 \text { articles) } \\
\text { Yeast (288 articles) } \\
\text { Yeast Genetics (135 articles) }\end{array}$ \\
\hline
\end{tabular}

